\title{
Prospective comparison between real time contrast enhanced and conventional ultrasound guidance in percutaneous biopsies of liver tumors.
}

\author{
Zeno Spârchez ${ }^{1,2}$, Pompilia Raduํ, Gabriel Kacso ${ }^{3}$, Mihaela Spârchez ${ }^{4}$, Teodor Zaharia ${ }^{1}$, \\ Nadim Al Hajjar 5
}

${ }^{1}$ Institute for Gastroenterology and Hepatology, ${ }^{2} 3 \mathrm{rd}$ Medical Department, ${ }^{3}$ Oncology Department, ${ }^{4}$ 2nd Pediatric Department, ${ }^{5} 3^{\text {rd }}$ Surgical Department, "Iuliu Haţieganu" University of Medicine and Pharmacy, Cluj-Napoca, Romania

\begin{abstract}
Aims: The performance of percutaneous echo-guided biopsy in the hepatic tumoral diagnosis is limited ( $90 \%$ sensitivity) by several factors, among which tumor characteristics (type, size, and location) play an important role. Contrast enhanced ultrasound guided percutaneous biopsy (CEUS-PLB) is a new developed technique aimed at increasing the accuracy of percutaneous biopsies. The objective of our study was to evaluate the feasibility and performance of CEUS as a guiding method in performing liver biopsy (PLB). Material and methods: One hundred and seventy one patients with liver tumors referred to the ultrasound department for LB were prospectively included in the study. PLB was performed with CEUS guidance in 90 patients (46 in patients with and 44 in patients without liver cirrhosis), in the remaining 81 (37 in patients with liver cirrhosis and 44 in patients without) with conventional US guidance. The lesions in the CEUS -PLB group were larger than those in the US-LB group (mean diameter $7.73 \mathrm{~cm}$ vs. $6.11 \mathrm{~cm}, \mathrm{p}>0.05$ ). In both groups the lesions were further divided in: a) lesions on cirrhosis; b) poorly visualized tumors; c) large tumors ( $>4 \mathrm{~cm})$; d) cystic tumors; e) recurrences after ablation; and f) portal vein thrombosis. Results: Real time CEUS-PLB was technically successful in 84 of the 86 procedures $(97.6 \%$ technical success rate). The rate of successful single puncture attempt in CEUS-PLB (43.02\%) was higher than in the US-PLB group $(23.4 \%)(p<0.05)$. The sensitivity of LB was significantly higher in the CEUS-PLB group than in the conventional US-LB group for all lesions $(96.5 \%$ vs. $81.48 \%, \mathrm{p}<0.05)$, for lesions on liver cirrhosis $(95.2 \%$ vs. $75 \%, \mathrm{p}<0.05)$, for large $(>6 \mathrm{~cm})$ $(97.8 \%$ vs. $82 \%, p<0.05)$, and for poorly visible lesions ( 100 vs. $66.6 \%, p=0.029)$. The patients with inconclusive pathological results after conventional guided LB were then biopsied with CEUS guidance. In all cases the final diagnosis could be established. One major complication occurred in each group $(\mathrm{p}>0.05)$. Conclusions: Percutaneous LB performed with CEUS guidance is a feasible and safe technique. It significantly improves the overall sensitivity of the procedure especially in patients with large lesions and in those poorly visualized on conventional ultrasound.
\end{abstract}

Keywords: hepatic tumors, percutaneous echoguided biopsy, contrast enhanced ultrasound

\section{Introduction}

In patients with liver tumors histopathological diagnosis by means of percutaneous biopsy is of great importance since it may provide crucial information for patient management, including the achievement of the final diagnosis, assessing prognosis, and selecting the proper

Received 22.09.2015 Accepted 21.10.2015

Med Ultrason

2015, Vol. 17, No 4, 456-463

Corresponding author: Zeno Spârchez, MD, Ph.D

Institute for Gastroenterology and Hepatology

Croitorilor 19-21, Cluj Napoca, Romania

Email: zsparchez@yahoo.co.uk therapy. The information offered by pathology is becoming even more important in the new era of molecular or genic oncological therapies.

In spite of a dramatic improvement in the imaging and tumoral markers diagnosis over the latter years, percutaneous needle biopsy is used frequently for the pathological diagnosis in oncology. Ultrasound is mostly used for guidance but with all the advantages offered by this imaging method, the overall sensitivity in the diagnosis of liver tumors remained around $90 \%$ [1-3].

In the past years improvements in percutaneous biopsy have emerged in terms of needle design [4] or ultrasound methods with a complementary role in guidance 
(color or Power Doppler, 3D/4D ultrasound, navigation systems, and contrast enhanced ultrasound, CEUS) [5$11]$.

Among these CEUS guidance has proved to be a feasible technique for increasing the efficacy of percutaneous biopsy [12]. CEUS has been used previously before percutaneous liver biopsy (PLB) to select non-necrotic areas or better locate/delineate the lesion as was successfully demonstrated in one large series [13]. Real time CEUS guided PLB was used to puncture invisible lesions in some small series $[10,11,14]$. To our knowledge no prospective study has been conducted to assess the effectiveness of "real time" CEUS guided biopsy in the diagnosis of liver tumors.

The aim of our study was to prospectively assess the technical feasibility and effectiveness of real time CEUS guided PLB in the diagnosis of liver tumors.

\section{Material and methods}

From January 2009 to December 2013, 175 patients (107 males, 68 females, aged from 20 to 90 years, mean 61.2 years) with liver tumors detected on conventional ultrasonography (B-US), contrast enhanced computed tomography (CT) or magnetic resonance imaging (MRI), with a high suspicion of malignancy were sent to our laboratory for PLB. The patients were randomly divided into 2 groups: a group who underwent PLB guided by conventional US (US-PLB) (81 patients) and another who underwent PLB guided by real time CEUS (CEUSPLB) (94 patients). In the US-PLB group there were 37 patients with lesions on normal liver and 44 patients on cirrhotic liver. In the CEUS-PLB group there were 48 patients with lesions on normal liver and 46 patients with lesions on cirrhotic liver.

Previous reports and studies [12-15] have identified several types of lesions that may benefit from CEUSPLB: large lesions, lesions poorly or invisible in conventional US, lesions with cystic content, residual or renascent tumors after percutaneous ablation and portal vein thrombosis. Both groups were balanced for these criteria.

The patients were informed about the benefits and risks of the procedure and they gave an informed consent. The study was approved by the Ethical Committee of the university.

\section{US-guided biopsy}

US-PLB was performed using a Aloka Prosound SSD $3500 \mathrm{SX}$ system with a $3.5 \mathrm{MHz}$ transducer in 31 patients and Logiq 7 system (General Electric, Milwaukee, USA) with a 3.5 3-5 MHz convex array wide band probe in 50 patients. Color Doppler examination was useful to avoid the hepatic artery branches and other major hepatic vessels.
For lesions in the left lobe a subxyphoid approach was used, while for those in the right lobe, an intercostal or right subcostal approach was necessary. The coagulation studies accepted for the hepatic tumor biopsy were a platelets count higher than $60000 / \mathrm{mmc}$ and prothrombin time less than $15 \mathrm{sec}$. All patients fasted for at least 8 hours before the procedure.

After localization of the lesion, with color-Doppler mapping, we chose an appropriate biopsy path avoiding the vascular structures. The biopsy was performed with real-time sonographic guidance using the free-hand technique. We used $1.2 \mathrm{~mm}$ diameter $(18 \mathrm{G})$ cutting needles (Bard) with a $1.4 \mathrm{~cm}$ long sampling notch coupled on a Biopty Gun (Bard, Cavington, USA). Before biopsy the skin was sterilized and local anesthesia with $2 \%$ lidocaine was performed. Special care was taken to avoid the direct puncture of superficial lesions; in such cases some effort was made to traverse some normal liver parenchyma. For deep-seated lesions the shortest pathway was used.

The number of puncture attempts was decided by the length and the aspect of the specimen sampled. If the first specimen sampled was judged to be unsatisfactory, a second attempt was made. The maximum number of punctures was 3. All patients were observed for 30 minutes after puncture and then sent to the gastroenterological department where they continued fasting for 2 hours. They remained in the hospital for the next 24 hours.

The tissue specimens were placed in $10 \%$ formaldehyde and then sent to the pathology department for histological examination by one experienced pathologist. Pathological diagnosis of hepatocellular carcinoma (HCC) was made according to the International Working Party criteria [16].

\section{CEUS-PLB}

Liver biopsies in this group were performed using a Logiq 7 machine (General Electric, Milwaukee, USA) with real-time contrast specific software and a 3-5 MHz convex array wide band probe.

Contrast enhanced imaging was performed according to the protocol used for the hepatic lesions examination $[17,18]$. Examination was performed with low acoustic power (mechanical index under 0.1) after injection of a SonoVue (BR1; Bracco, Milan, Italy). SonoVue consisted of sulfur-hexafluoride (SF6) vapor-filled and phospholipid-stabilized microbubbles with a diameter uniformly smaller than $8 \mu \mathrm{m}$; these microbubbles circulate in the intravascular space crossing pulmonary and systemic capillary circulation. In each patient $2.4 \mathrm{~mL}$ of contrast-agent were administered. The low mechanical index technique avoids destruction of bubbles thus allowing the identification of the entire vascular phase of 
contrast agent perfusion, consisting of the arterial phase (15-30 s after injection of agent), portal phase (30-60 s after injection of agent), and late parenchymal phase [5]. CEUS-PLB was done in a split-screen mode, which displays the CEUS image on the right side and the background B-mode US image on the left side, simultaneously, on a single monitor. The mechanical index was usually set to 0.1 . Focus was positioned at the bottom of the screen to minimize microbubble destruction. Field of view and gain were optimized to provide the clearest depiction of the lesion.

After the target lesion was selected with the use of conventional US and the results obtained from previous imaging studies (CT, MRI) the skin was sterilized and the predicted needle path was anesthetized with $2 \%$ lidocaine.

Prior to the intravenous injection of a $2.4 \mathrm{ml}$ SonoVue, the needle is inserted into the skin entry at the level selected. When the lesion began to clearly appear following the contrast agent injection, the needle was advanced, via an intercostal or subcostal approach. In cases of large lesions well depicted on conventional US the needle was directed in the arterial phase into the enhanced, perfused areas. If the specimen was considered unsatisfactory a second puncture was performed in the late arterial or portal phase. For poorly visible tumoral lesions in conventional US the biopsy was performed: a) in the arterial phase if the lesion had been well delineated in this phase as a hyper or hypovascular area; b) in the parenchymal phase if the lesion was known/suspected to be a metastases and was not clearly depicted by conventional US. The lesion was punctured when it washed out and the tissue to lesion contrast ratio was maximal; and c) in both phases usually if the specimen sampled in arterial phase was judged to be unsatisfactory.

If the lesion had a CEUS aspect typical for a benign tumor (like haemangioma or focal nodular hyperplasia) and/or the lesion's size was small, the puncture was avoided.

All the CEUS examinations and CEUS-PLB as well as the US-PLB were performed by the same physician (ZS) with high experience in interventional ultrasound.

\section{Final lesion diagnosis}

If the pathological diagnosis was positive for malignancy the result was considered true positive. In case of a certain benign tumor (haemangioma or focal nodular hyperplasia) the result was also considered true positive. In cases when the result of the biopsy was negative for malignancy or benignity (regenerative nodule, abscess) the final diagnosis was established by a combination of clinical, biochemical (tumoral markers) and imaging data and/or a follow up of 6 months. If malignancy was later confirmed, the result of the biopsy was considered false negative. In some patients from the US-PLB group the biopsy was repeated using CEUS guidance.

\section{Statistical analysis}

The difference in diagnostic accuracy between the 2 groups was calculated with the chi-square test using the SPSS version 10. Enumeration data were given as mean $\pm \mathrm{SD}$ and were analyzed with an unpaired $t$ test. A p value of less than 0.05 was considered significant.

\section{Results}

In 4 patients CEUS performed immediately before the CEUS-PLB revealed typical pattern of haemangioma (3 lesions) and focal nodular hyperplasia (1 lesion). In those cases the biopsy was not performed and the patients were not included in the study.

The characteristics of patients in both groups are presented in table I.

There was no statistically significant difference between the 2 groups in the size ranges of the liver lesions $(\mathrm{p}>0.05)$.

Real time CEUS-PLB was technically successful in 84 of the 86 procedures $(97.6 \%$ technical success rate). In 2 cases the lesion could be punctured neither in ar-

Table I. Characteristics of the lesions in the study group

\begin{tabular}{|c|c|c|c|}
\hline & CEUS-PLB & US-PLB & $\begin{array}{l}\text { Signifi- } \\
\text { cance }\end{array}$ \\
\hline Number & 86 & 81 & NS \\
\hline Age (mean, range) & $62.07(31-90)$ & $60.3(20-76)$ & NS \\
\hline $\operatorname{Sex}(F / M)$ & $30 / 56$ & $34 / 47$ & NS \\
\hline Malignant/benign & $79 / 7$ & $76 / 5$ & NS \\
\hline Cirrhosis & 42 & 44 & NS \\
\hline Size $($ mean \pm SD $)$ & $7.73 \pm 11.03$ & $6.11 \pm 10.96$ & NS \\
\hline$<1 \mathrm{~cm}$ & 0 & 0 & $\mathrm{NC}$ \\
\hline $1-2 \mathrm{~cm}$ & 7 & 5 & $\mathrm{NC}$ \\
\hline $2-4 \mathrm{~cm}$ & 15 & 18 & NS \\
\hline $4-6 \mathrm{~cm}$ & 11 & 13 & NS \\
\hline$>6 \mathrm{~cm}$ & 47 & 39 & NS \\
\hline \multicolumn{4}{|l|}{ Lesion type } \\
\hline Large lesions & 53 & 52 & NS \\
\hline $\begin{array}{l}\text { Poorly visualized } \\
\text { lesions }\end{array}$ & $18^{*}$ & 15 & NS \\
\hline Cystic lesions & 4 & 2 & $\mathrm{NC}$ \\
\hline $\begin{array}{l}\text { Residual/renascent } \\
\text { tumors after ablation }\end{array}$ & 4 & 3 & $\mathrm{NC}$ \\
\hline PVT & 6 & 6 & NS \\
\hline Other & $1 * *$ & $3 * * *$ & $\mathrm{NC}$ \\
\hline
\end{tabular}

*2 invisible lesions, $* *$ low enhancing lesion in $\mathrm{CT}, * * *$ lesions that couldn't be included in neither of these types; NC not calculated (small sample size); NS - not significant; CEUS-PLB - contrast enhanced ultrasound guided percutaneous biopsy, US-PLB - ultrasound guided percutaneous biopsy. 
terial nor in portal or parenchymal phase. These cases were considered false negative results. In one case the lesion could not be correctly targeted in the arterial phase but was in the parenchymal phase (fig 1). Twenty-four CEUS-PLBs were performed only in the arterial phase, 13 in the parenchymal phase and 49 in both phases. The indications for CEUS-PLB and the pathological results are presented in Table II. The US-PLB was technically successful in all cases.

The average number of puncture attempts in USPLB and CEUS-PLB groups was 1.80 and 1.65 respectively (difference not significant). The rate of a successful single puncture attempt in CEUS-PLB (37 of $86,43.02 \%$ ) was higher than in the US-PLB group $(18$ of $81,23.4 \%)\left(p<0.05, \chi^{2}=6.354\right)$. The frequency of benign lesions was $8.13 \%$ in the CEUS-PLB group and $6.17 \%$ in the US-PLB group (difference not significant).

In the CEUS-PLB group a final pathological result could be reached in 83 of 86 patients ( $96.5 \%$ accuracy). The accuracy is significantly higher than for US-PLB,

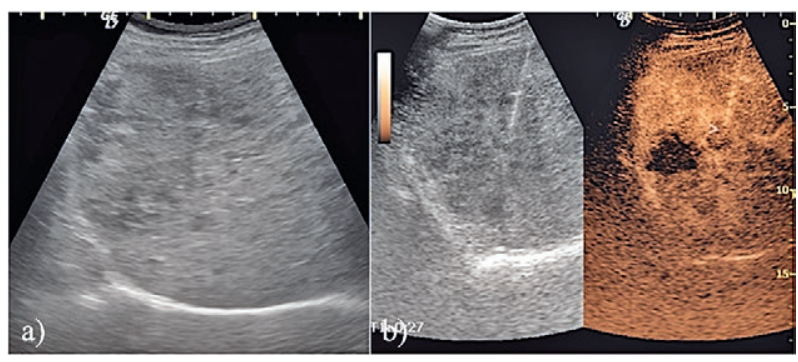

Fig 1. a) Large hepatic tumor in a 62 old male with liver cirrhosis; b) in arterial phase a necrotic area $(>)$ was mistargeted instead of an enhanced area. The punctured performed in the portal phase revealed a hepatocellular carcinoma. which has led to a correct pathological diagnosis in 66 of 81 lesions $(81.48 \%)\left(p<0.01, \chi^{2}=8.281\right)$. The difference is statistically significant for lesions on liver cirrhosis $\left(95.2 \%\right.$ vs. $\left.75 \%, p<0.05 \chi^{2}=5.576\right)$ but not for lesions occurring in the liver without cirrhosis $(97.7 \%$ vs. $91.9 \%$, $p=0.48, \chi^{2}=0.499$ ) (fig 2).

For different sizes of the lesions the accuracies are depicted in figure 3. It should be mentioned that although CEUS-PLB was superior to US-PLB for all size ranges and also for portal thrombus biopsy, the difference is statistically significant only for tumors larger than $6 \mathrm{~cm}\left(97.8 \%\right.$ vs. $\left.82 \%, \mathrm{P}=0.03, \chi^{2}=4.521\right)$ (fig 4$)$ and those poorly visible on US (100 vs. $66.6 \%, p=0.029$, $\chi^{2}=4.732$ ) (fig 5). For local recurrences after percutaneous ablation the accuracy of CEUS-PLB was superior to US-PLB (100 \% vs. 66.6\%) but without statistical significance $(p=0.87)$. Fifteen patients in the US-PLB group and 3 patients in the CEUS-PLB group had false negative results (Table III).

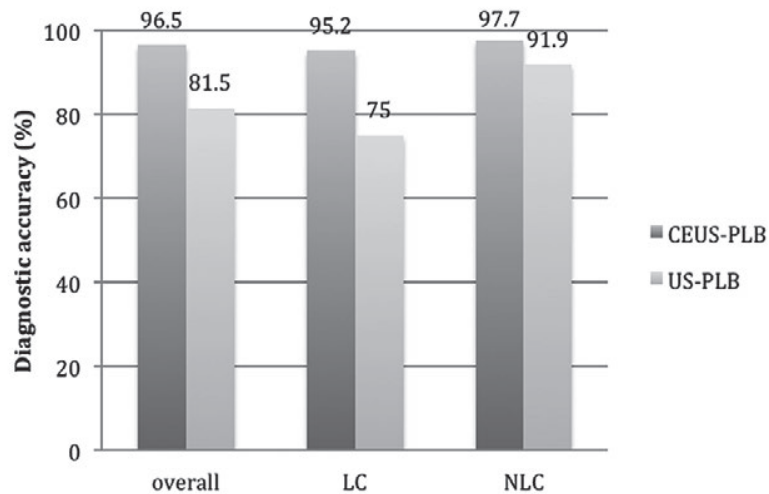

Fig 2. Diagnostic accuracy of US and CEUS-PLB (LC liver cirrhosis, NLC, non cirrhotic liver)

Table II. Vascular phases of the liver where CEUS-PLB were performed with correspondent indications and pathological results

\begin{tabular}{lllll}
\hline CEUS-PLB & Indications to perform CEUS-PLB & No. & Pathologic results & No. \\
\hline Arterial phase & Large lesions & 18 & Hepatocellular carcinoma & 9 \\
24 lesions & Poorly visible lesions (hypervascular on CT) & 2 & Hypervascular metastasis & 5 \\
& Local recidives after ablation & 2 & Cholangiocarcinoma & 5 \\
& PVT & 1 & Abscesses & 2 \\
& Hypovascular unclear lesion on CT & 1 & Benign (fibrosis, trombosed haemangioma) & 3 \\
Parenchymal & Poorly visible lesions & 9 & Metastasis (colon, pancreas) & 6 \\
phase & Large lesions ${ }^{1}$ & 3 & Cholangiocarcinoma & 5 \\
13 lesions & PVT $^{1}$ & 1 & Regenerative nodules & 1 \\
& & & Malignant PVT (HCC) & 1 \\
Arterial and pa- & Large lesions & 32 & Hepatocellular carcinoma \\
renchymal phase & Poorly visible lesions & 7 & Metastatic carcinoma (mostly adenocarcinoma) & 16 \\
49 lesions & PVT & 4 & Cholangiocarcinoma & 8 \\
& Cystic lesions & 4 & Hypervascular metastasis (neuroendocrine, GIST) & 4 \\
& Local recidives after ablation & 2 & Lymphoma & 1 \\
& & & Cirrhosis (false negative results) & 3 \\
\hline
\end{tabular}

${ }^{1}$ The PLB could not be performed in the arterial phase, HCC hepatocellular carcinoma, PVT portal vein thrombosis. 


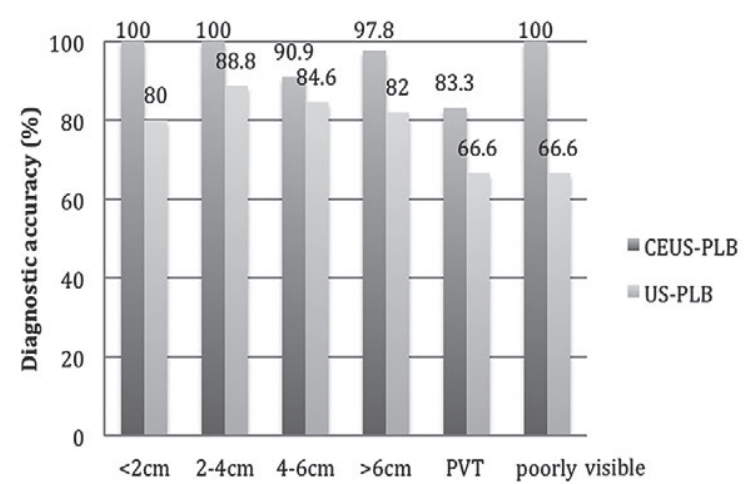

Fig 3. Accuracies of US and CEUS-PLB for different tumor sizes and types

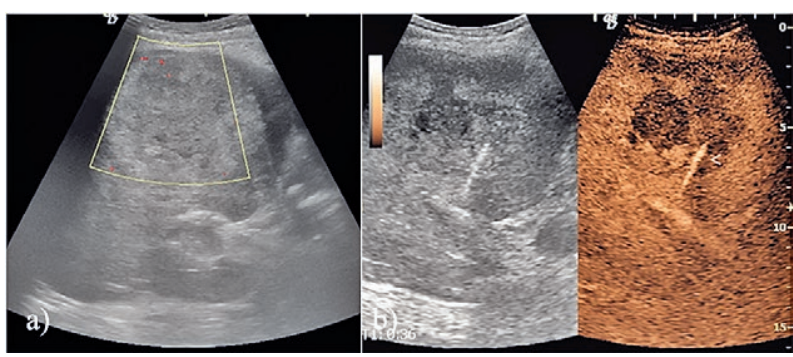

Fig 4. Large hepatic tumor in a 63 year old male with liver cirrhosis: a) Power Doppler showed very few vessels within the lesion; b) CEUS guided liver biopsy with split mode screen. The specimen was sampled from an enhanced marginal area avoiding the central necrosis. The distal, active part of the needle is seen $(>)$. Pathological diagnosis was hepatocellular carcinoma.

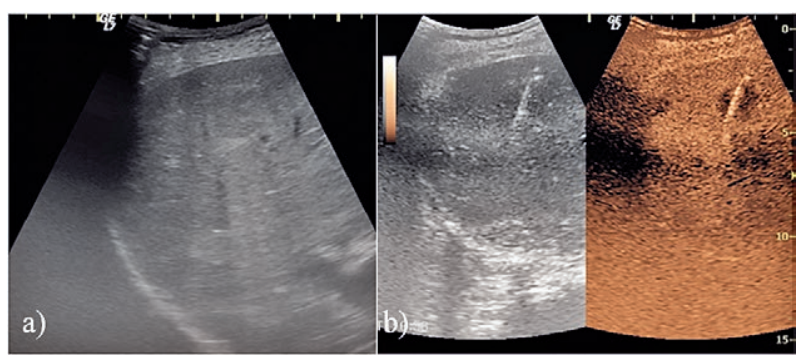

Fig 5. a) Inhomogenous parenchyma without clear lesions in a 67 female patient with colic adenocarcinoma; b) In the parenchymal phase several metastasis were depicted and one, $1.5 \mathrm{~cm}$ in size, was punctured $(>)$ with CEUS guidance.
Finally 10 of the 15 patients with false negative results were re-biopsied using CEUS-PLB. In all cases the final diagnosis could be achieved (HCC 4 patients, malignant PVT 2 patients, metastatic adenocarcinoma 4 patients).

The frequency of major complications was similar in both groups $(1.23 \%$ and $1.11 \%$ respectively). One patient in US-PLB developed an uncontrolled intraperitoneal bleeding requiring surgery. In the CEUS-PLB group one intraperitoneal bleeding managed conservatively occurred.

\section{Discussion}

PLB is used extensively to diagnose hepatic tumors in clinical practice. Despite all the gained experience, improvements in needles and US techniques, false negative results still persist. There are several factors that may be responsible for this: a) the presence of tumoral necrosis or fatty changes usually found in large lesions. Necrotic tissue cannot be identified on B-mode ultrasound, especially before liquefaction has occurred; b) small, deep located (in posterior segments of the liver) or lesions in risky locations (i.e. near vascular structures, gallbladder, and colon) may be difficult to visualize and target with B mode US; c) invisible or poorly visible lesions in B mode ultrasound like small liver metastasis or isoechoic HCCs require several passages to target the lesions. In such cases contrast enhanced imaging methods such as $\mathrm{CT}$ and MRI are required to image and target the lesion $[2,3]$.

In the last years CEUS has emerged not only as a valuable diagnosis method for focal liver lesions (in terms of characterization and detection) but also as a guidance method for various liver interventions including percutaneous biopsy [10-14,18-22].

Previous studies have demonstrated that CEUS guidance may improve the accuracy of liver biopsy in large hepatic tumors with consistent necrosis, hypovascular tumors, complex cystic masses, lesions invisible or poorly visible to conventional ultrasound, and in diagnosis of PVT [9-15,23]. In these studies CEUS was used before

Table III. False negative result on US and CEUS-PLB

\begin{tabular}{lllll}
\hline False negative results & US-PLB & No. & CEUS-PLB & No. \\
\hline Liver cirrhosis & Necrosis in large lesions* & 6 & Targeting error & 2 \\
& Poorly visualized lesions & 3 & Large regenerative nodule with HCC foci & 1 \\
& Small lesions & 1 & Total & $\mathbf{3}$ \\
& PVT & 2 & & \\
Ton-cirrhotic liver & Total & $\mathbf{1 2}$ & & \\
& Poorly visualized lesions & 2 & & \\
& Small lesion & 1 & & \\
& Total & $\mathbf{3}$ & & \\
\hline
\end{tabular}

*including one patient with recidive after percutaneous ablation, HCC- hepatocellular carcinoma, PVT portal vein thrombosis 
PLB [13] or as real time guidance for lesions not visible at conventional US $[11,14]$. To our knowledge this is the first prospectively comparative study assessing the usefulness of real time CEUS guided biopsy in liver tumors in comparison with conventional US guided biopsy.

Some technical difficulties have been reported with real-time CEUS biopsy: a) sometimes the biopsy needle cannot be reliably identified in the bright contrast enhancement in the surrounding parenchyma which may lead to misplacement [14]; b) the short period of arterial enhancement available for puncture. This difficulty is present mostly for lesions not depicted in B mode US and depicted only in the arterial phase of CEUS as hyperenhancing areas. All these limitations can be overcome by using: a) a split-screen mode which displays the CEUS image on one side and the B mode US image on the other side (available now on most of the systems) with a better conspicuity of the needle in B mode image; b) smaller amounts of contrast with less enhancement in the arterial phase and c) needle guides [11,12,14]. A preprocedural injection may aid in the detection of the lesion and planning the biopsy [13].

The results of our study showed that real-time CEUSPLB is a feasible technique with a $97.6 \%$ success rate. Two other similar studies have reported lower success rates, $84 \%$ and $91.6 \%$ respectively $[11,14]$. In those studies only lesions not seen on the B mode US were included whereas in our study only 2 out of 86 lesions were not seen on B mode US.

The present study has demonstrated that the overall accuracy of CEUS-PLB is significantly superior to USPLB. Although CEUS-PLB has provided increased accuracies for all subgroups, the difference was statistically significantly only for lesions occurring in liver cirrhosis, for those larger than $6 \mathrm{~cm}$, and for B mode US poorly visible lesions.

The overall accuracy of CEUS-PLB was similar to that found in the study of $\mathrm{Wu}$ et al $(95.3 \%)$ who used CEUS before biopsy to obtain intralesional information regarding the presence and disposition of the viable tumoral tissue [13]. In the same study an increased accuracy was demonstrated in the CEUS group for all size subgroups, the values obtained being quite similar to our study. A significant difference was found only for lesions less than $2 \mathrm{~cm}$ [13]. In our study although the difference in accuracy for lesions less than $2 \mathrm{~cm}$ was important, it did not reach statistical significance presumably due to the low number of cases.

The significantly increased accuracy for CEUS-PLB in lesions $>6 \mathrm{~cm}$ may be explained by the possibility to target the needle in an enhanced part of the tumor, thus avoiding necrosis, usually present in large lesions, mainly in the central part $[8,12,13]$.
The superiority of CEUS-PLB for poorly visible/invisible lesions was also demonstrated in 2 previous studies $[11,14]$. In a prospective study that evaluated percutaneous real-time CEUS guided biopsy of focal hepatic lesions not confidently localized on B-mode US in 44 patients, Yoon et al reported an overall sensitivity in the diagnosis of malignancy of $88 \%$ [14].

CEUS-PLB was also superior to US-PLB in patients with neoplastic PVT, results that were previously demonstrated by our group [23]. The false negative result present in our study was due to a targeting error. Based on the results of previous studies we may state that when confirmation of malignant thrombosis is required, PVT biopsy may be performed by CEUS guidance to reduce false-negative results due to blind sampling [23,24].

Local recurrences after percutaneous ablation for HCC or metastases in this study were more accurately diagnosed using CEUS-PLB. Recurrences are located around the treated area, often are large and cannot be differentiated from the treated, necrotic area using $\mathrm{B}$ mode US [13]. In practice, contrast enhanced CT, MRI, and CEUS have high sensitivities and accuracy for the detection of the residual tumor [25-27]. However, there are some circumstances where the imaging methods are less reliable (such as perilesional arterio-portal fistulas or other vascularization disturbances, diffuse HCC in the vicinity of the ablated area) or the pattern of recurrence (diffuse or multicentric tumors) requires the start of a systemic therapy (e.g. with Sorafenib) [27,28]. In such cases a biopsy may be required.

The present study revealed the fact that the advantage of CEUS-PLB over US-PLB is far more evident for lesions in the cirrhotic liver in comparison with the liver without cirrhosis. Possible explanations are the difficulties to detect and target lesions in the cirrhotic liver, which are more often poorly visible, diffuse, with intravascular spread, or large. In fact the false negative results of USPLB in cirrhotic patients were due to necrosis in $50 \%$ of cases and to poorly visualized lesions in $25 \%$. However, these findings should be validated in a prospective large trial with a significant number of patients.

The use of CEUS guidance did not increase the complications rate. Moreover, having a higher rate of successful single puncture attempts, it may lead to lower complications respectively with classical US guided biopsy.

By integrating the results of the present study with the experience gained from previous similar studies, an important question may arise. What is recommended before a liver biopsy? To do a CEUS before biopsy and select cases where CEUS guidance may help or to perform real time CEUS-PLB in all cases? The answer depends 
on several factors including the population referred for biopsy. In an oncological population where the diagnosis was done by CT/MRI, a CEUS guided PLB would be more appropriate. For lesions detected by US, CEUS performed immediately thereafter will further characterize the lesion to avoid unnecessary biopsy and will offer important information for choosing a CEUS-PLB (presence of necrotic areas, complex or less demarcated lesion).

This study has some limitations. First, the selection of patients and the inclusion in one of the subgroups (i.e. poorly visible lesions) depended on the subjective judgment of one physician. Second, nearly $40 \%$ of the biopsies in the US-PLB group were performed using a middle range US system, with less image resolution, which may have contributed to the lower accuracy for this group. However, the physician who performed all the biopsies had considerable experience on that system which has resulted in a high confidence in his skills. Thirdly, the same physician who performed also the CEUS guided biopsies had a 15 years experience with US guided biopsy and 5 years experience with liver CEUS studies. In this setting the results of this study may not be generalized to other smaller centers with less experienced physicians.

In conclusion, CEUS guided biopsy is a feasible and low risk technique that increases the diagnosis accuracy in liver tumors. Some particular tumor subtypes (large, poorly visible, or on cirrhotic liver) may benefit the most from this technique.

Conflict of interest: none

Acknowledgement The work is part of the research project within BIOPTICON 2329/2008 PNCDI II 20072013.

\section{References}

1. Francque SM, De Pauw FF, Van den Steen GH, Van Marck EA, Pelckmans PA, Michielsen PP. Biopsy of focal liver lesions: guidelines, comparison of techniques and costanalysis. Acta Gastroenterol Belg 2003; 66: 160-165.

2. Mircea P, Pop S, Chira R, Valean S, et al. Echoguide biopsy in abdominal tumors. Factors influencing overall results, other than the type and size of the biopsy needle. Rev Rom Ultrasonografie 2001; 3: 39-47.

3. Solbiati L, Ierace T, Tonolini M, Cova L. Guidance and monitoring of radiofrequency liver tumor ablation with contrast-enhanced ultrasound. Eur J Radiol 2004; 51: S19S23.

4. Diederich S, Padge B, Vossas U, Hake R, Eidt S. Application of a single needle type for all image-guided biopsies: results of 100 consecutive core biopsies in various organs using a novel tri-axial, end-cut needle. Cancer Imaging 2006; 6: 43-50.
5. Lencioni R, Caramella D, Bartolozzi C. Percutaneous biopsy of liver tumors with color Doppler US guidance. Abdom Imaging 1995; 20: 206-208.

6. Polaków J, Janica J, Serwatka W, Ladny JR, ZukowskaSerwatka K. Value of three-dimensional sonography in biopsy of focal liver lesions. J Hepatobiliary Pancreat Surg 2003; 10: 87-89.

7. Birth M, Iblher P, Hildebrand P, Nolde J, Bruch HP. Ultrasound-guided interventions using magnetic field navigation. First experiences with Ultra-Guide 2000 under operative conditions. Ultraschall Med 2003; 24: 90-95.

8. Bang N, Bachmann Nielsen M, Vejborg I, Mellon Mogensen A. Clinical report: contrast enhancement of tumor perfusion as a guidance for biopsy. Eur J Ultrasound 2000; 12: $159-161$.

9. Skjoldbye B, Pedersen MH, Struckmann J, Burcharth F, Larsen T. Improved detection and biopsy of solid liver lesions using pulse-inversion ultrasound scanning and contrast agent infusion. Ultrasound Med Biol 2002; 28: 439444.

10. Meuwly JY, Schnyder P, Gudinchet F, Denys AL. Pulseinversion harmonic imaging improves lesion conspicuity during US-guided biopsy. J Vasc Interv Radiol 2003; 14: 335-341.

11. Schlottmann K, Klebl F, Zorger N, Feuerbach S, Schölmerich J. Contrast-enhanced ultrasound allows for interventions of hepatic lesions which are invisible on conventional B-mode. Z Gastroenterol 2004; 42: 303-310.

12. Sparchez Z, Radu P, Zaharia T, et al. Usefulness of contrast enhanced ultrasound guidance in percutaneous biopsies of liver tumors. J Gastrointestin Liver Dis 2011; 20: 191-196.

13. $\mathrm{Wu} \mathrm{W}$, Chen $\mathrm{MH}$, Yin SS, et al. The role of contrast-enhanced sonography of focal liver lesions before percutaneous biopsy. AJR Am J Roentgenol 2006; 187: 752-761.

14. Yoon SH, Lee KH, Kim SY, et al. Real-time contrast-enhanced ultrasound-guided biopsy of focal hepatic lesions not localised on B-mode ultrasound. Eur Radiol 2010; 20: 2047-2056.

15. Grossjohann HS, Bachmann Nielsen M. Ultrasound contrast agents may help in avoiding necrotic areas at biopsy. Ultraschall Med 2006; 27: 2-3.

16. International Working Party. Terminology of nodular hepatocellular lesions. Hepatology 1995; 22: 983-993.

17. Claudon M, Dietrich CF, Choi BI, et al. Guidelines and Good Clinical Practice Recommendations for Contrast Enhanced Ultrasound (CEUS) in the Liver - Update 2012: A WFUMB-EFSUMB Initiative in Cooperation with Representatives of AFSUMB, AIUM, ASUM, FLAUS and ICUS. Ultrasound Med Biol 2013; 39: 187-210.

18. Strobel D, Seitz K, Blank W, et al. Contrast-enhanced ultrasound for the characterization of focal liver lesions--diagnostic accuracy in clinical practice (DEGUM multicenter trial). Ultraschall Med 2008; 29: 499-505.

19. Sporea I, Badea R, Popescu A, et al. Contrast-enhanced ultrasound (CEUS) for the evaluation of focal liver lesions - a prospective multicenter study of its usefulness in clinical practice. Ultraschall Med 2014; 35: 259-266. 
20. Chen MH, Yang W, Yan K, et al. The role of contrast-enhanced ultrasound in planning treatment protocols for hepatocellular carcinoma before radiofrequency ablation. Clin Radiol 2007; 62: 752-760.

21. Miyamoto N, Hiramatsu K, Tsuchiya K, Sato Y. Contrastenhanced sonography-guided radiofrequency ablation for the local recurrence of previously treated hepatocellular carcinoma undetected by B-mode sonography. J Clin Ultrasound 2010; 38: 339-345.

22. Chan AK, Hegarty C, Klass D, et al. The Role of Contrastenhanced Ultrasound in Guiding Radiofrequency Ablation of Hepatocellular Carcinoma: A Retrospective Study. Can Assoc Radiol J 2015; 66: 171-178.

23. Sparchez Z, Radu P, Zaharia T, et al. B-mode and contrast enhanced ultrasound guided biopsy of portal vein thrombosis. Value in the diagnosis of occult hepatocellular carcinoma in liver cirrhosis. Med Ultrason 2010; 12: 286-294.

24. Sorrentino P, D'Angelo S, Tarantino L, Ferbo U, Bracigliano A, Vecchione R. Contrast enhanced sonography versus biopsy for the differential diagnosis of thrombosis in hepatocellular carcinoma patients. World J Gastroenterol 2009; 15: 2245-2251.

25. Sparchez Z, Radu P, Anton O, Socaciu M, Badea R. Contrast enhanced ultrasound in assessing therapeutic response in ablative treatments of hepatocellular carcinoma. J Gastrointestin Liver Dis 2009; 18: 243-248.

26. Lu MD, Yu XL, Li AH, et al. Comparison of contrast enhanced ultrasound and contrast enhanced CT or MRI in monitoring percutaneous thermal ablation procedure in patients with hepatocellular carcinoma: a multi-center study in China. Ultrasound Med Biol. 2007; 33: 1736-1749.

27. Zheng SG, Xu HX, Lu MD, et al. Role of contrast-enhanced ultrasound in follow-up assessment after ablation for hepatocellular carcinoma. World J Gastroenterol 2013; 19: 855-865.

28. Reynolds AR, Furlan A, Fetzer DT, et al. Infiltrative hepatocellular carcinoma: what radiologists need to know. Radiographics 2015; 35: 371-386. 